\title{
Classification of high-grade endometrium carcinomas using molecular and immunohistochemical methods
}

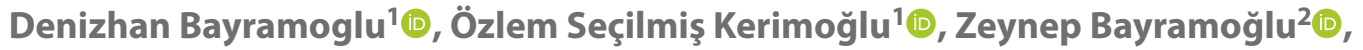

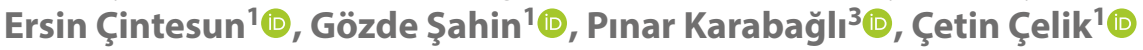 \\ ${ }^{1}$ Department of Obstetrics and Gynecology, Faculty of Medicine, Selcuk University, Konya, Turkey \\ ${ }^{2}$ Department od Pathology Konya Education and Research Hospital, Konya Education and Research Hospital, Konya, Turkey \\ ${ }^{3}$ Selcuk University, Department of Pathology, Konya, Turkey
}

\begin{abstract}
Objectives: As a result of the integration of molecular changes into the histological classification of cancers, which increases diagnostic repeatability, the differences between the groups become more prominent and targeted therapies gain significance. The most comprehensive molecular study regarding endometrial carcinomas (EC) is The Cancer Genome Atlas (TCGA) project. According to TCGA, endometrial carcinomas are classified into four molecular prognostic subgroups: copy-number-low/p53-wild-type (p53wt), DNA polymerase epsilon (POLE)-mutated/ultramutated (POLEmt), microsatellite-instability/hypermutated (MSI), and copy-number-high/p53-mutated (p53mt). In this study, we aim to apply the molecular classification to our high-grade endometrial cancer patients, and particularly, to identify our overtreated patients.
\end{abstract}

Material and methods: Ninety-seven patients diagnosed with high-grade EC in Selcuk University, Faculty of Medicine between 2009-2018 were retrospectively evaluated and classified into four subgroups. Primary outcomes of overall and progression-free survival were evaluated for clinical, pathological, and molecular features. Further, all molecular groups were divided into endometroid and non-endometrioid groups, and disease-free survival (DFS) and overall survival (OS) were investigated across groups.

Results: According to molecular classification, 23 patients (23.7\%) were assigned to the MSI group, 21 (21.6\%) to the POLEmt group, $40(41.2 \%)$ to the p53mt group, and $13(13.4 \%)$ to the $p 53 w t$ group. Patients' $D F S(p=0.001)$ and OS rates $(p=0.001)$ were significantly different according to their molecular classification. The results of our analyses determined that, in the molecular classification of high-grade ECs, the p53mt group had the poorest prognosis and the POLEmt group had the best prognosis. Tumor size, myometrial invasion, lymphovascular space invasion (LVSI), lymph node metastasis, cervical invasion, ovarian invasion and stage showed statistically significant differences based on molecular classification $(p<0.05)$.

Conclusions: The use of molecular classification in the clinical practice will allow more accurate prognostic prediction and more appropriate treatment planning, particularly as high-grade ECs constitute a heterogenous group with poor prognosis. Key words: endometrial cancer; high-grade; MMR gene; molecular classification; POLE; prognosis; p53

Ginekologia Polska 2023; 94, 1: 3-11

\section{INTRODUCTION}

Endometrial carcinomas (EC) is the most common gynecological cancer in the western world. In the last decade, both its incidence and the associated mortality demonstrated an increase [1]. Causes of such an unfavorable trend probably lie in an inaccurate risk stratification, which would cause many patients to be undertreated or overtreated [2].

In 2013, the study conducted by TCGA Research Network classified endometrial carcinomas into four molecular prognostic subgroups using the methods of whole genome sequencing, exome sequencing, microsatellite analysis, and copy number analysis, as follows: POLEmt, microsatellite instability (MSI), p53wt and p53mt [3-6]. Accordingly, recent studies have investigated methodologies that can be clinically applied to identify these groups. These methodologies can also target the underlying molecular abnormalities and influence the prognosis $[4,7-10]$.

According to the work by TCGA, high-grade ECs (grade 3, endometrioid, serous and clear cell) are distributed across all four molecular groups. This project stressed that supple-

\footnotetext{
Corresponding author:

Özlem Seçilmiş Kerimoğlu

Department of Obstetrics and Gynecology, Faculty of Medicine, Selcuk University, Konya, Turkey

e-mail: ozlemsecilmis@hotmail.com
} 
menting histomorphology with the molecular characteristics would increase the accuracy of the treatment approach. However, the methods used here are too costly to be implemented at every center. Therefore, simpler and more applicable molecular-based classification methods are being investigated. A high-grade is an unfavorable prognostic factor for endometrial cancer. However, some high-grade cases manifest a better prognosis. The identification of cases with favorable and poor prognoses in a molecular study would influence the prognosis and the treatment modalities.

In clinical practice, there exists a patient group with low-grade EC that shows early recurrence and short survival rates, and, in contrast, there also exists a patient group with high-grade EC that does not show recurrence and presents longer survival rates. However, the high-grade EC group with no recurrence and longer survival rates is encountered more commonly than the former. Thus, in this study, we aim to molecularly classify the high-grade endometrial carcinoma cases in Turkey and investigate whether molecular classification is a useable method for the determination of the patients' prognoses.

\section{MATIERIAL AND METHODS}

Paraffin blocks and hematoxylin-eosin-stained preparations of 97 patients diagnosed with high-grade EC who were operated in the Gynecological Oncology Department of Selçuk University, Faculty of Medicine between 20092018 were retrieved from the pathology archives. Demographic and clinical data of the patients were obtained from the hospital system, files and via telephone.

Patients' age, tumor size, myometrial invasion, LVSI, lymph node metastasis, cervical invasion, ovarian invasion, International Federation of Gynecology and Obstetrics (FIGO) stage, histological subtype, operation date, follow-up duration, DFS and OS data were recorded. High-grade ECs were accepted as grade 3 , endometrioid, serous and clear cell carcinomas. The study has been conducted by the principles of the Helsinki Declaration and approved by the Institutional Review Board (No:2019/26 Date:16.01.2019).

\section{Immunohistochemical Analysis}

Sections obtained from paraffin blocks were immunohistochemically stained for p53, MLH-1, MSH-2, MSH- 6 and PMS-2 according to the standard procedure using a DAKO Omnis autostainer (Agilent, Santa Clara, CA 95051, USA). Immunohistochemistry was performed on paraffin-embedded specimens. The samples were fixed in $10 \%$ neutral formalin, dehydrated in alcohol, embedded in paraffin, and serial sections of $5 \mu \mathrm{m}$ were prepared on slides. Following rehydration, samples were put in $0.01 \mathrm{M}$ citrate buffer $(\mathrm{pH}=6)$ and were warmed twice in a microwave oven for five minutes every time at $750 \mathrm{~W}$ for recovery of antigens. After cool- ing for 20 minutes at room temperature, the sections were washed with phosphate buffer saline (PBS). To eliminate endogenous peroxidase activity, sections were maintained in $3 \%$ hydrogen peroxide for 20 minutes and thereafter washed with PBS. Subsequently, sections were incubated in blocking serum for 10 minutes to block non-specific binding. Sections were incubated with primary monoclonal antibodies against MLH1 (clone ES05 diluted 1:50, DAKO), PMS2 (clone EP51 diluted 1:40, DAKO), MSH2 (clone FE1 1 diluted 1:50, DAKO), MSH6 (clone EP49 diluted 1:50, DAKO), p53(clone DO-7 diluted 1:50 DAKO) for $30 \mathrm{~min}$. Negative control sections were treated with nonimmune serum diluted in the same fashion. Labeling was performed using the Universal LSAB kit (Dako, Carpinteria, CA, USA) as per the manufacturer's instructions. Staining was completed with DAB Chromogen (DBS, Pleasanton, CA, USA) for 1-2 minutes, and slides were counterstained with Harris's hematoxylin, dehydrated and then cover-slipped with permount. All specimens were examined with a light microscope.

All immunohistochemical staining analyses included control tissues. Mutation-type p53 staining was accepted as overexpression (intense nuclear staining in $>75 \%$ tumor cells) or absence of nuclear expression.

\section{Pole mutation analysis protocol}

For POLE mutations, analyses specific to the P286R/S, V411L, F367S/C/L, P436R, A456P, L424P, E396Q, S279A, F367 mutations were performed using the Sanger sequence. Pole mutation analysis protocol:

1. The human Pole gene, which consists of approximately 3000 bases, was studied by dividing it into two groups as 1400 and 600 bases, considering the possibility of being fractured due to the DNAs being isolated from paraffinic samples.

2. Primer sequences and PCR conditions used for both sites are given below:

POLE-1_F: GGTGTTCAGGGAGGCCTAAT

POLE-1_R: CCCATGAGATGTGGTGACAG

POLE-2_F: GGTGCCTGTTAGGAACTTGC

POLE-2_R: GCTCCATGGGAATAATGGTG

- $95^{\circ} \mathrm{C} 5$ minutes - initial denaturation

- 40 cycles:

- $95^{\circ} \mathrm{C}$ for 45 seconds - denaturation

- $57^{\circ} \mathrm{C}$ for 45 seconds - annealing

- $72{ }^{\circ} \mathrm{C}$ for 60 seconds - extension

- $72{ }^{\circ} \mathrm{C}$ for 5 minutes - final extension

- The temperature was dropped to $4^{\circ} \mathrm{C}$ and PCR was completed.

The amplification results obtained by PCR (kyratec thermocycler) were carried out in $1.5 \%$ agarose gel prepared with $1 \times$ TAE buffer in 100-volt current for 90 minutes in electrophoresis and imaged under UV light using ethidium 
bromide dye. PCR reaction was performed with Solis Biodyne (Estonia) FIREPol ${ }^{\circledR}$ DNA Polymerase Taq polymerase enzyme. It was observed that the PCR process was successful by obtaining a single band in agarose gel after PCR for your samples.

3. In the PCR product purification stage, the "HighPrepTM PCR Clean-up System" (AC-60005) purification enzyme was used for the obtained single band samples and purified according to the kit procedures.

4. For Sanger Sequencing samples, ABI 3730XL Sanger sequencer (Applied Biosystems, Foster City, CA) and BigDye Terminator v3.1 Cycle Sequencing Kit were used in Macrogen's Netherlands laboratory (Applied Biosystems, Foster City, CA).

5. The case was included in the POLE ultra-mutation group in the presence of any of these mutations.

\section{Determination of molecular subgroups}

In order to divide high-grade ECs into TCGA-like molecular subgroups, all patients were analyzed by immunohistochemistry for MLH-1, MSH-2, MSH-6 and PMS-2, due to the higher usability in daily life and the lower cost. All the re- maining patients were screened for POLE hotspot mutations (P286R/S, V411L, F367S/C/L, P436R, A456P, L424P, E396Q, $\mathrm{S} 279 \mathrm{~A}, \mathrm{~F} 367)$ using the Sanger sequencing method. The case was included in the POLEmt group if one of these mutations were detected. For the remaining patients, p53 staining was performed for immunohistochemical analysis. p53 mutants were included in the p53mt group. Meanwhile, our p53-wild patients were included in the p53wt group.

\section{Statistical analyses}

Relationships between categorical variables were tested using the chi-square test. The Kaplan Meier method was used for the prediction, and the Log rank test for the comparison of survival times. Cox Regression analysis was used in the determination of factors that influence overall survival. Analyses were performed using the SPSS for Windows 22.0 software. $\mathrm{P}<0.05$ was considered significant.

\section{RESULTS}

A total of 97 patients were included in the study. A patient who was included in the MMR group as a result of immunohistochemical staining is shown in Figure 1. Based
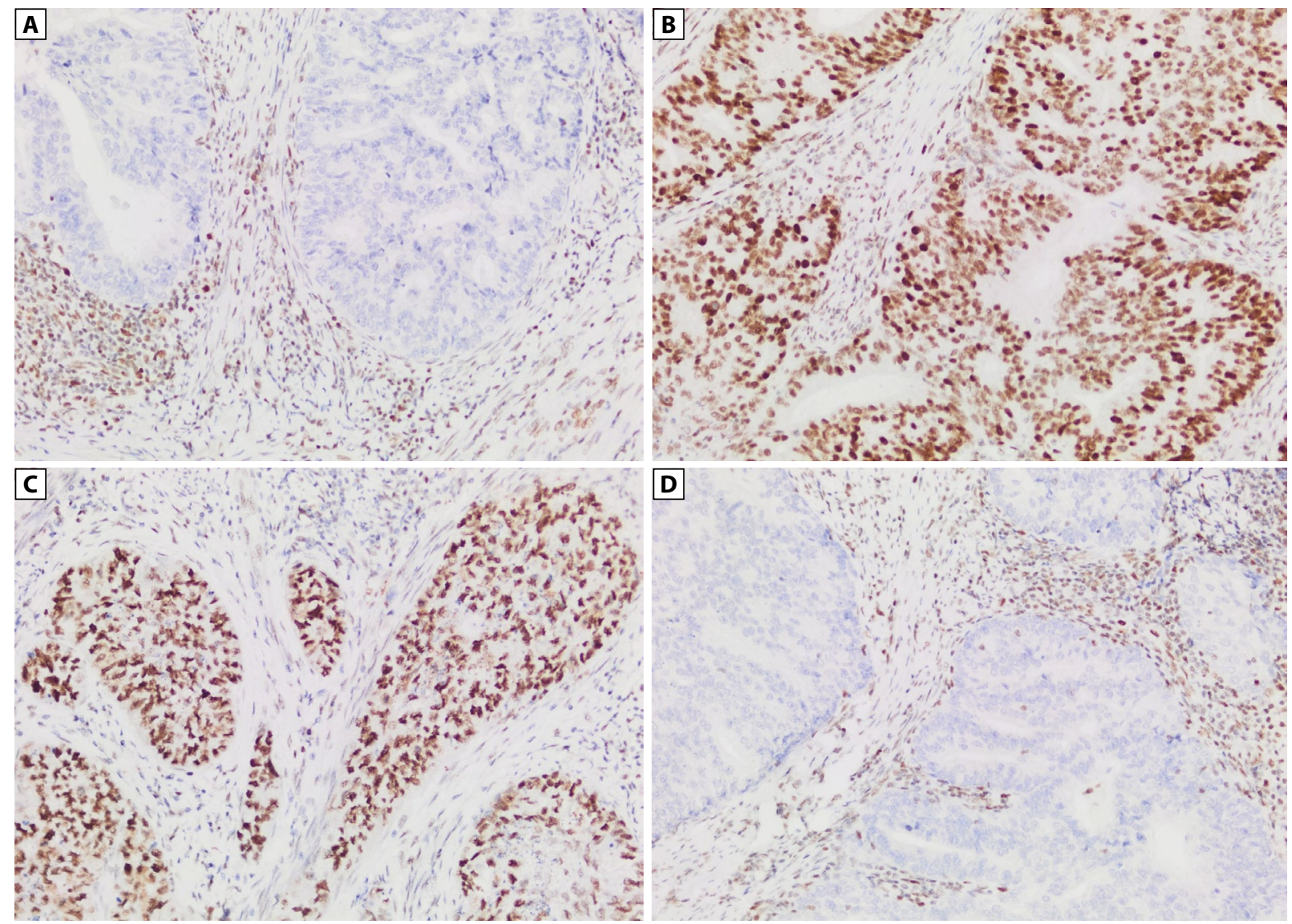

Figure 1. Use of immunohistochemistry for molecular MMR subgoup; A. High grade endometrial carcinoma with loss of MLH1 expression; B. Nuclear staining was observed in the stroma and tumor cells with MSH2 (normal staining); $\mathbf{C}$. Nuclear staining was observed in the stroma and tumor cells with MSH6 (normal staining); D. High grade endometrial carcinoma with loss of PMS2 expression 
Table 1. Descriptive statistics, demografic, clinicopathological, and molecular parameters within the four molecular groups

\begin{tabular}{|c|c|c|c|c|c|c|c|}
\hline & & $\begin{array}{c}\text { TOTAL } \\
n=97(100 \%)\end{array}$ & $\begin{array}{c}\text { POLEmt } \\
\mathrm{n}=\mathbf{2 1}(21.6 \%)\end{array}$ & $\begin{array}{c}\text { MSI } \\
n=23(23.7 \%)\end{array}$ & $\begin{array}{c}\text { P53wt } \\
n=13(13.4 \%)\end{array}$ & $\begin{array}{c}\text { P53mt } \\
n=40(41.2 \%)\end{array}$ & $p$ value \\
\hline \multirow{2}{*}{ Age } & $<60$ & 27 (27.8\%) & $7(33.3 \%)$ & $9(39.1 \%)$ & $5(38.5 \%)$ & $6(15.0 \%)$ & \multirow{2}{*}{0.122} \\
\hline & $\geq 60$ & 70 (72.2\%) & 14 (66.7\%) & 14 (60.9\%) & 8 (61.5\%) & 34 (85.0\%) & \\
\hline \multirow{3}{*}{$\begin{array}{l}\text { Histological } \\
\text { subtype }\end{array}$} & Endometioid & $33(34 \%)$ & $10(47.6 \%)$ & 10 (43.5\%) & $4(30.8 \%)$ & 9 (22.5\%) & \multirow{3}{*}{0.099} \\
\hline & Serous & $58(59.8 \%)$ & $9(42.9 \%)$ & $13(56.5 \%)$ & 9 (69.2\%) & $27(67.5 \%)$ & \\
\hline & Clear cell & $6(6.2 \%)$ & $2(9.5 \%)$ & 0 & 0 & $4(10.0 \%)$ & \\
\hline \multirow{2}{*}{ Tumor size } & $<2 \mathrm{~cm}$ & $7(7.2 \%)$ & $2(9.5 \%)$ & $5(21.7 \%)$ & 0 & 0 & \multirow{2}{*}{$0.005^{*}$} \\
\hline & $\geq 2 \mathrm{~cm}$ & 90 (92.8\%) & $19(90.5 \%)$ & $18(78.3 \%)$ & $13(100.0 \%)$ & $40(100.0 \%)$ & \\
\hline \multirow{2}{*}{$\begin{array}{l}\text { Myometrial } \\
\text { invasion }\end{array}$} & $<50 \%$ & 49 (50.5\%) & $8(38.1 \%)$ & 17 (73.9\%) & $12(92.3 \%)$ & $12(30.0 \%)$ & \multirow{2}{*}{$0.001^{*}$} \\
\hline & $>50 \%$ & 48 (49.5\%) & $13(61.9 \%)$ & $6(26.1 \%)$ & $1(7.7 \%)$ & $28(70.0 \%)$ & \\
\hline \multirow{2}{*}{$\begin{array}{l}\text { Lymphovascular } \\
\text { invasion }\end{array}$} & Yes & 45 (46.4\%) & $10(47.6 \%)$ & $4(17.4 \%)$ & $6(46.2 \%)$ & $25(62.5 \%)$ & \multirow{2}{*}{$0.008^{*}$} \\
\hline & No & $52(53.6 \%)$ & 11 (52.4\%) & 19 (82.6\%) & $7(53.8 \%)$ & $15(37.5 \%)$ & \\
\hline \multirow{2}{*}{$\begin{array}{l}\text { Lymph nodal } \\
\text { metastasisi }\end{array}$} & Yes & $38(36.8 \%)$ & $7(33.3 \%)$ & $4(17.4 \%)$ & $1(7.7 \%)$ & $26(65.0 \%)$ & \multirow{2}{*}{$0.001^{*}$} \\
\hline & No & $59(57.2 \%)$ & $14(66.7 \%)$ & 19 (82.6\%) & $12(92.3 \%)$ & $14(35.0 \%)$ & \\
\hline \multirow{2}{*}{$\begin{array}{l}\text { Cervical } \\
\text { invasion }\end{array}$} & Yes & 34 (35.1\%) & $6(28.6 \%)$ & $2(8.7 \%)$ & $1(7.7 \%)$ & 25 (62.5\%) & \multirow{2}{*}{$0.001^{*}$} \\
\hline & No & 63 (64.9\%) & 15 (71.4\%) & $21(91.3 \%)$ & $12(92.3 \%)$ & $15(37.5 \%)$ & \\
\hline \multirow{2}{*}{ Ovary invasion } & Yes & $20(20.6 \%)$ & $1(4.8 \%)$ & $2(8.7 \%)$ & 0 & $17(42.5 \%)$ & \multirow{2}{*}{$0.001^{*}$} \\
\hline & No & 77 (79.4\%) & 20 (95.2\%) & 21 (91.3\%) & $13(100.0 \%)$ & $23(57.5 \%)$ & \\
\hline \multirow{2}{*}{$\begin{array}{l}\text { Omentum } \\
\text { invasion }\end{array}$} & Yes & $14(14.4 \%)$ & $2(9.5 \%)$ & $2(8.7 \%)$ & 0 & $10(25.0 \%)$ & \multirow{2}{*}{0.077} \\
\hline & No & $83(85.6 \%)$ & $19(90.5 \%)$ & 21 (91.3\%) & $13(100.0 \%)$ & 30 (75.0\%) & \\
\hline \multirow{2}{*}{ Stage } & $1-2$ & 52 (56.4\%) & 12 (57.1\%) & 17 (73.9\%) & $12(92.3 \%)$ & $11(27.5 \%)$ & \multirow{2}{*}{$0.001^{*}$} \\
\hline & $3-4$ & 45 (43.6\%) & 9 (42.9\%) & 6 (26.1\%) & $1(7.7 \%)$ & 29 (72.5\%) & \\
\hline
\end{tabular}

MSI — microsatellite instability

on the results of the performed analyses, 21 patients (21.6\%) were classified into the POLEmt group, 23 patients (23.7\%) into the MSI group 13 (13.4\%) into the p53wt group, and $40(41.2 \%)$ into the p53mt group.

The 33 patients who were in the endometrioid group based on histological classification, 10 (30.3\%) were classified into the POLEmt group, 10 (30.3\%) into the MSI group, $4(12.1 \%)$ into the p53wt group, and 9 (27.3\%) into the p53mt group according to the novel classification. On the other hand, of the 64 patients in the non-endometrioid group, 11 (17.2\%) were classified into the POLEmt group, $13(20.3 \%)$ into the MSI group, $9(14.1 \%)$ into the p53wt group, and 31 (48.4\%) into the p53mt group according to the novel classification.

The 21 patients who were placed in the POLE ultra-mutated group based on the novel classification, 10 (47.6\%) had the endometrioid subtype, 11 had the non-endometrioid subtype ( 9 (42.9\%) serous, 2 (9.5\%) clear cell). Of the 23 patients who were placed in the MSI group based on the novel classification, 10 (43.5\%) had the endometrioid subtype, $13(56.5 \%)$ had the non-endometrioid subtype (all serous). Of the 11 patients who were placed in the p53wt group based on the novel classification, 4 (30.8\%) had the endometrioid subtype, $9(69.2 \%)$ had the non-endometrioid sub- type (all serous). Of the 40 patients who were placed in the p53mt group based on the novel classification, 9 (22.5\%) had the endometrioid subtype, 31 had the non-endometrioid subtype (27 (67.5\%) serous, 4 (10.0\%) clear cell).

The 21 patients in the POLEmt group, 19 showed the P436R hotspot mutation. Only two patients showed both V411L and P436R hotspot mutations. Pathological and clinical characteristics of all our patients are summarized in Table 1. Tumor size, myometrial invasion, LVSI, lymph node metastasis, cervical invasion, ovarian invasion, and stage had a statistically significant relationship with the molecular classification. Tumor size was determined to be larger than $2 \mathrm{~cm}$ at a high rate across all groups $(p=0.005)$. Myometrial invasion was more than $1 / 2$ at a high rate in the $p 53 \mathrm{mt}$ and POLEmt groups $(p=0.001)$. LVSI, lymph node metastasis, cervical invasion, ovarian invasion, and stage were higher in the p53mt group, with statistical significance. Age, histological type, ovarian invasion did not have a statistically significant relationship with the molecular groups.

The mean follow-up time was 60 months. Across all patients, OS was calculated as $94,498(84,5-104,4)$ months and DFS as 92.505 (81.9-103) months. Three-year and five-year DFS rates were calculated as $72.4 \%$ and $61 \%$; 


\begin{tabular}{|c|c|c|c|c|c|}
\hline \multirow{3}{*}{ Molecular classification } & \multicolumn{4}{|c|}{ Mean } & \multirow{3}{*}{ p value } \\
\hline & \multirow{2}{*}{ Estimate } & \multirow{2}{*}{ Std. error } & \multicolumn{2}{|c|}{ 95\% Confidence interval } & \\
\hline & & & Lower bound & Upper bound & \\
\hline Pole ultramutated & 109.250 & 3.655 & 102.086 & 116.414 & \multirow{5}{*}{$0.001^{*}$} \\
\hline MSI hyper mutated & 96.688 & 9.444 & 78.178 & 115.198 & \\
\hline Copy number high & 62.728 & 8.397 & 46.270 & 79.186 & \\
\hline Copy number low & 106.778 & 11.215 & 84.797 & 128.759 & \\
\hline Overall & 92.505 & 5.362 & 81.995 & 103.015 & \\
\hline
\end{tabular}

MSI - microsatellite instability

\begin{tabular}{|c|c|c|c|c|c|}
\hline \multirow{3}{*}{ Molecular classification } & \multicolumn{4}{|c|}{ Mean } & \multirow{3}{*}{ p value } \\
\hline & \multirow{2}{*}{ Estimate } & \multirow{2}{*}{ Std. error } & \multicolumn{2}{|c|}{$\mathbf{9 5 \%}$ Confidence interval } & \\
\hline & & & Lower bound & Upper bound & \\
\hline Pole ultramutated & 109.250 & 3.655 & 102.086 & 116.414 & \multirow{5}{*}{$0.001^{*}$} \\
\hline MSI hyper mutated & 97.724 & 9.297 & 79.502 & 115.946 & \\
\hline Copy number high & 69.013 & 8.025 & 53.283 & 84.742 & \\
\hline Copy number low & 107.939 & 10.913 & 86.549 & 129.330 & \\
\hline Overall & 94.498 & 5.074 & 84.552 & 104.443 & \\
\hline
\end{tabular}

MSI - microsatellite instability

three-year and five-year OS rates were calculated as $76.9 \%$ and $60.4 \%$, respectively, for all patients.

DFS and OS outcomes by molecular groups are summarised in Tables 2 and 3. The Long-Rank test was used to evaluate the prognostic capacity of our molecular classification (Fig. 2). We divided each molecular group into histological subtype (endometrium and non-endometrioid) and examined whether there was a prognostic difference or not (Fig. 3). However, we could not find statistically significant (Tab.4). Based on the performed analyses, the POLEmt group showed the best survival and the p53mt group the poorest survival.

\section{DISCUSSION}

EC is the sixth most commonly occurring cancer in the women and the 15th most common cancer overall [11]. Despite this, molecular-based research and clinical progress regarding EC is indisputably slow when compared with other cancers. The reason for this could be that more than $75 \%$ of the women diagnosed with EC are diagnosed at an early stage (stages 1-2) and show five-year survival rates of $75-90 \%$. However, in patients with recurrent and advanced-stage $\mathrm{EC}$, response to chemotherapy is low and the clinical outcomes are quite poor [10-13]. ECs have been classified based on histomorphology since 1983. Although immunohistochemical methods are used by expert gynecopathologists, particularly in the classification of high-grade EC, inconsistencies have been observed [10-13].
Furthermore, consistency across pathologists is also weak for morphological risk factors such as grade and LVSI [14]. Therefore, a more reliable classification that will integrate clinical, morphological and molecular characteristics is needed for adequate and effective treatment $[15,16]$.

Objective estimation of the risk group and the prognosis based on the molecular classification of curettage materials allows treatment options such as pre-operative targeted therapy, neoadjuvant chemotherapy, and fertility sparing surgery in young patients [10].

Based on the numerous studies in the literature, it was shown that about $25 \%$ of tumors classified by pathologists as high-grade endometrioid tumors as a result of molecular classification showed frequent p53 mutations and had a molecular phenotype resembling uterine serous carcinoma. Thus, this striking similarity between endometrioid tumors and uterine serous tumors suggests that genomic-based classification would allow better management of the patients' treatment. Our aim is to achieve a better classification of endometrial carcinomas with the use of histopathological and molecular findings and minimize the 'overtreated' and'undertreated'patients based on the new classification. An example we can provide is the case that patients who are evaluated as serous type based on histological classification and are treated accordingly, are categorized as POLEmt based on molecular classification. As observed in many series, including the present study, the POLEmt group is associated with a much more favorable prognosis. It is 
A

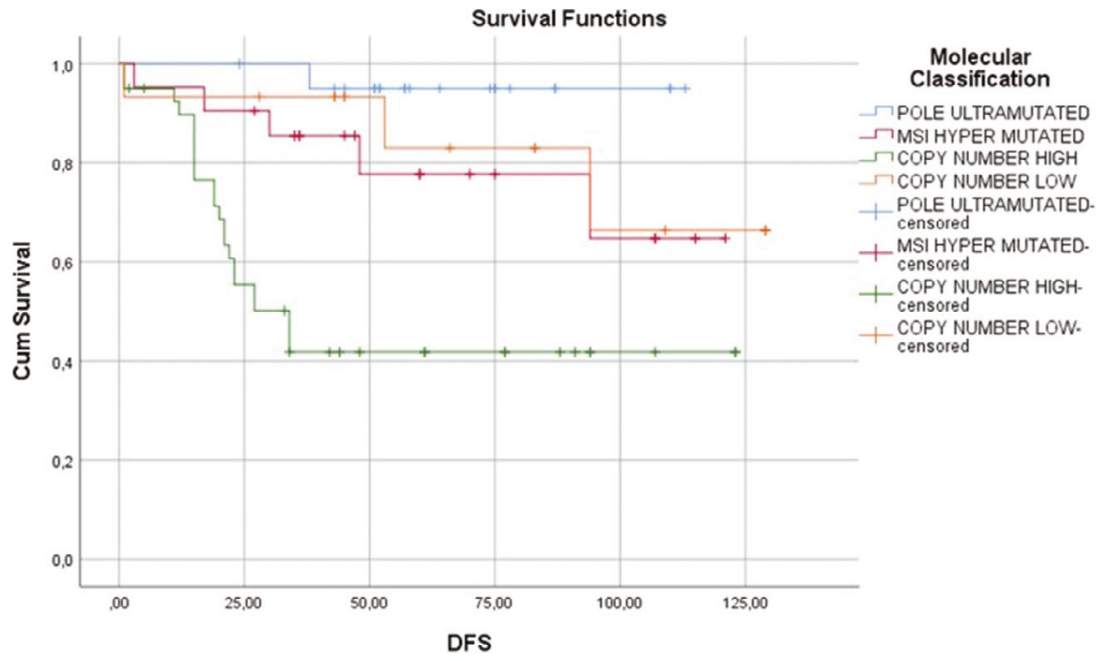

B

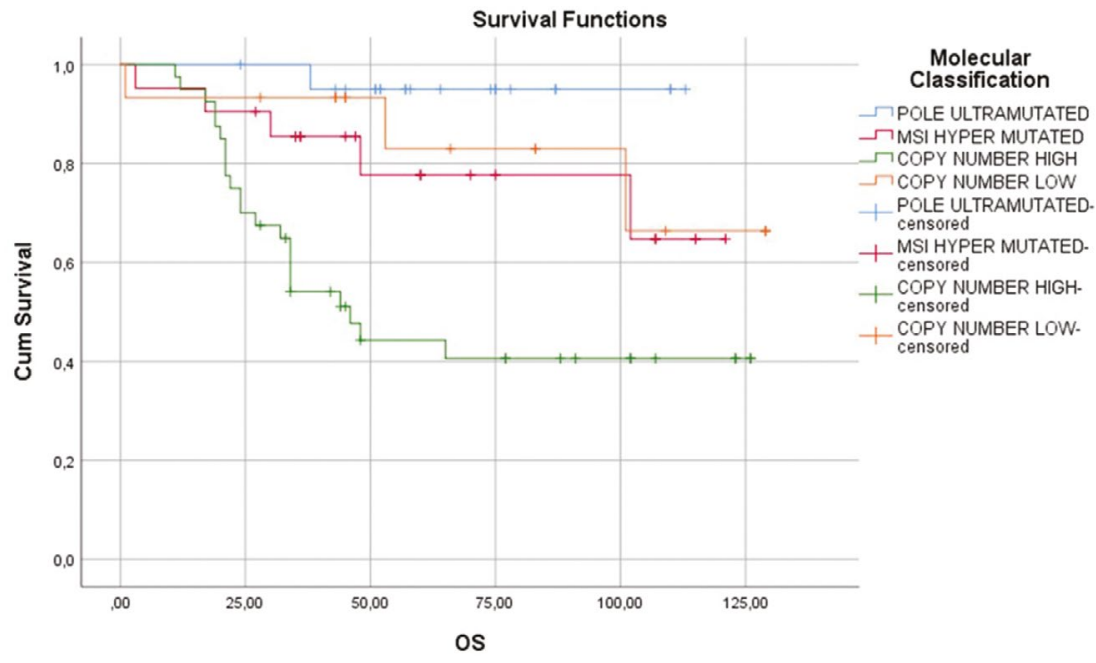

Figure 2. Kaplan-Meier plots assessed by long-rank test to evaluate; Disease free survival (A) and overall survival for new molecular groups (B); DFS - disease-free survival; OS - overall survival

not yet known if this is due to the administration of the aggressive treatment or whether it is independent from the treatment. Perhaps, the POLE ultra-mutation phenotype is more responsive to treatment or, as has been suggested previously, has a higher immune infiltrate that may be further stimulated by introduction of treatment(s) [17]. However, if these patients have a perfect prognosis regardless of the treatment, it is possible that a toxic treatment has been administered with no survival benefit, exposing them to unnecessary side effects, causing psychological difficulties, and generating unnecessary expenses and workload.

Recent studies have provided more information suggesting that in endometrial carcinomas involving POLE mutations, the course of the disease is ameliorated by the simulation of the immune system via the T-lymphocytes of the patients, influencing the clinical outcomes [18]. Endometrial carcinomas harboring loss-of-function mutations in
POLE are associated with a highly favorable prognosis, as seen in the present study.

Exome sequencing data from colorectal carcinoma and endometrial carcinomas that have somatic POLE exonuclease domain mutations show that the coding regions of these tumors alone have acquired about 5000 somatic bases. Compared with cancers without exonuclease domain mutations, all base types are higher in frequency. The discovery of a new colorectal carcinoma and endometrial carcinoma type based on POLE mutations increases the likelihood of similar discoveries in the future [19].

Although molecular classification is highly significant regarding the prognosis, it is associated with various problems. One of these is the possibility that changes associated with multiple molecular subtypes will be detected in a single tumor. This rate was determined as 3.5\% across all studies [20]. A portion of POLEmt and MSI tumors were also deter- 
A

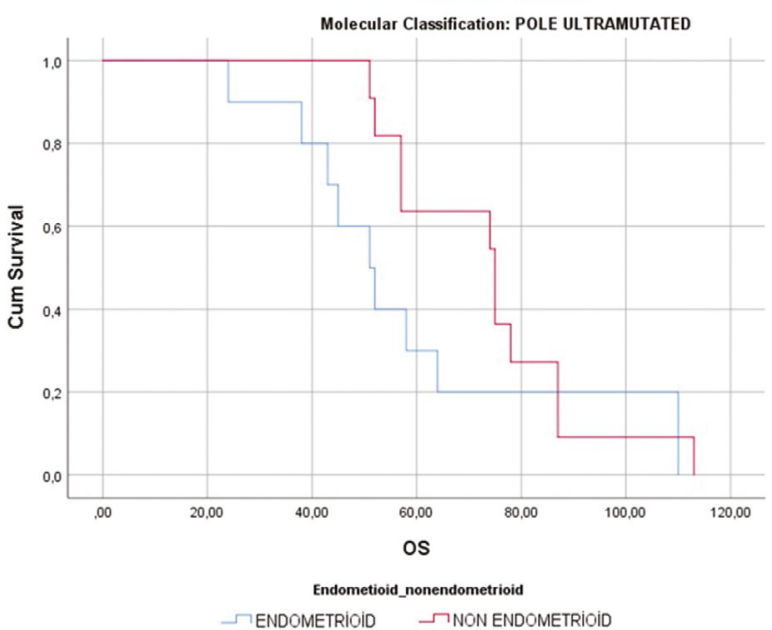

C

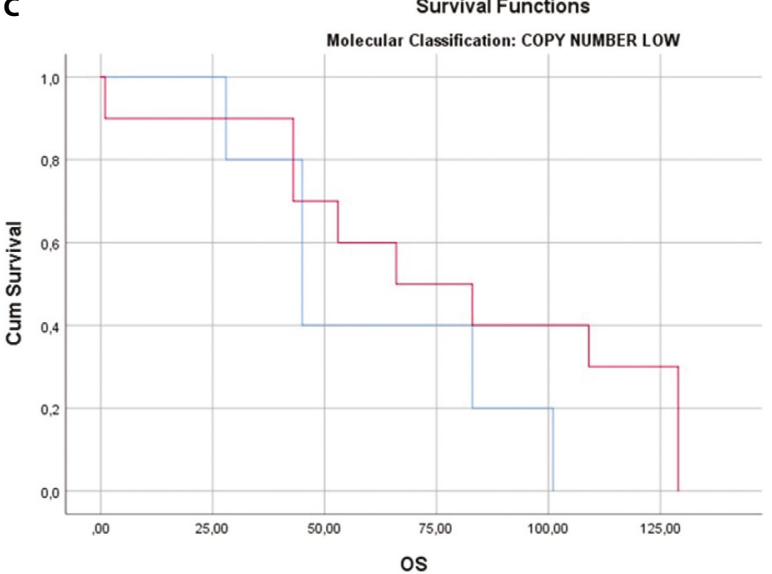

Endometioid_nonendometrioic

GENDOMETRIOID $\neg$ NON ENDOMETRIOID
B

Survival Functions

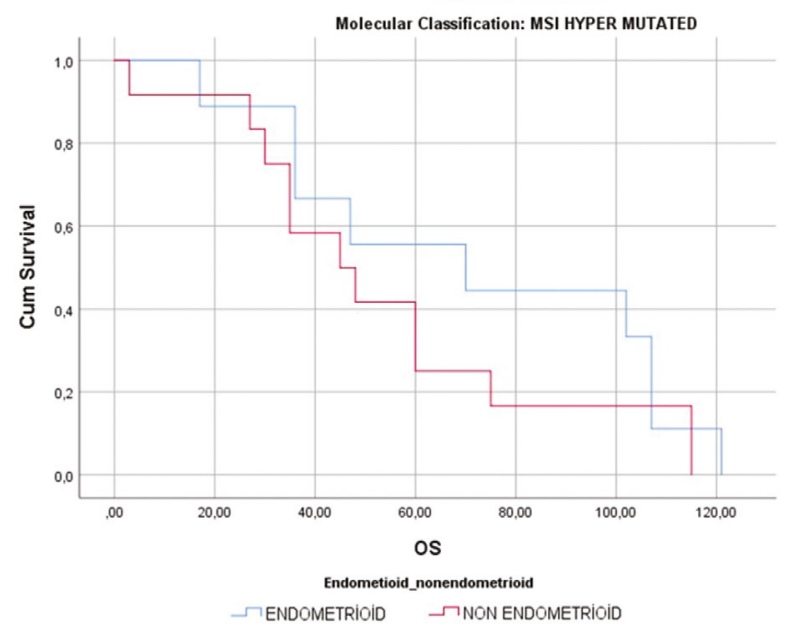

D

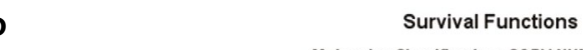

Molecular Classification: COPY NUMBER HIGH

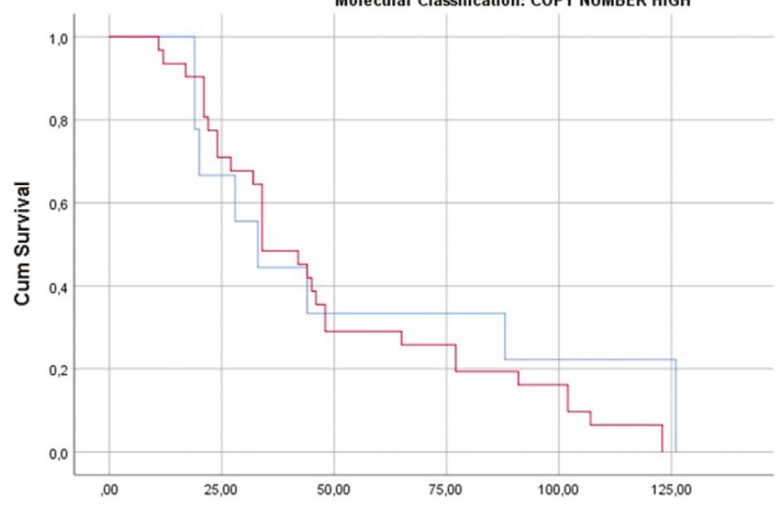

os

Endometioid_nonendometrioid

$\neg$ ENDOMETRIOID $\rightarrow$ NON ENDOMETRIOID

Figure 3. Overall survival of molecular groups according to endometrioid and non-endometrioid histological subtypes; A. POLEmt group; B. Microsatellite instability (MSI) group; C. p53wt group D) p53mt group; OS — overall survival

\begin{tabular}{|c|c|c|c|c|c|c|}
\hline \multirow{3}{*}{$\begin{array}{l}\text { Molecular } \\
\text { subtype }\end{array}$} & \multirow{3}{*}{ Histological subtype } & \multicolumn{4}{|c|}{ Mean } & \multirow{3}{*}{ p value } \\
\hline & & \multirow{2}{*}{ Estimate } & \multirow{2}{*}{ Std. error } & \multicolumn{2}{|c|}{$95 \%$ Confidence interval } & \\
\hline & & & & Lower bound & Upper bound & \\
\hline \multirow{2}{*}{ POLEmt } & Endometrioid & 59.500 & 9.102 & 41.660 & 77.340 & \multirow{2}{*}{0.223} \\
\hline & Non-Endometrioid & 73.273 & 5.610 & 62.277 & 84.269 & \\
\hline \multirow{2}{*}{ MSI } & Endometrioid & 71.444 & 12.907 & 46.147 & 96.742 & \multirow{2}{*}{0.365} \\
\hline & Non-Endometrioid & 54.000 & 9.809 & 34.775 & 73.225 & \\
\hline \multirow{2}{*}{ P53wt } & Endometrioid & 60.400 & 13.571 & 33.802 & 86.998 & \multirow{2}{*}{0.237} \\
\hline & Non-Endometrioid & 78.500 & 14.116 & 50.832 & 106.168 & \\
\hline \multirow{2}{*}{ P53mt } & Endometrioid & 55.889 & 15.053 & 26.385 & 85.393 & \multirow{2}{*}{0.423} \\
\hline & Non-Endometrioid & 49.742 & 6.017 & 37.948 & 61.536 & \\
\hline
\end{tabular}

MSI - microsatellite instability 
mined to have $\mathrm{p} 53$ mutations. Such cases pose the problem of differential diagnosis from serous-like, copy-number high group. Reliable evaluation of tumors of such characteristics is not possible due to the low number of cases and insufficient clinical data. Also, since studies predominantly include serous and high-grade endometrioid-type cases, the role of molecular study is uncertain for less common histotypes. A study conducted on this matter on dedifferentiated, undifferentiated carcinomas demonstrated frequent switch sucrose non-fermentable (SWI/SNF) protein loss in these tumors, which was not included in the TCGA classification [21]. Certainly, the classification needs to be elucidated by further studies that will be conducted on these matters.

In our study, the p53mt group had markedly the poorest prognosis, in line with the pioneering TCGA and other studies $[3,9,10]$. Our study also verified the prognostic value of molecular groups. We further divided each group as endometrioid or non-endometrioid and investigated whether there were prognostic differences between these subgroups. However, since we had a low number of patients in each molecular group and we performed this study on only high-grade endometrial cancer patients, we did not determine a statistically significant difference between the endometrioid and non-endometrioid subgroups. A metanalysis conducted by Travaglino and colleagues investigated a total of 2818 patients and all molecular groups were divided into endometrioid and non-endometrioid subgroups to study OS. This metanalysis determined the best prognosis for the POLEmt endometrioid group. Meanwhile, the group with the poorest prognosis was the MSI non-endometrioid group [22]. In fact, this situation is quite significant. The prognoses could be evaluated more successfully when the patients' molecular findings and histopathological findings were evaluated in combination.

A study that performed only molecular classification of high-grade endometrial carcinomas classified $9 \%$ of the patients into the POLEmt group, 32\% into the MMR group, 18\% into the p53wt group and 39\% into the p53mt group (17). In the present study, we classified $21.6 \%$ of our patients into the POLEmt, $23.7 \%$ into the MSI, $13.4 \%$ into the p53wt, and $41.2 \%$ into the $553 \mathrm{mt}$ group.

It has been emphasized in the literature that POLEmt patients are seen at younger ages [5, 23]. POLEmt patients were reported to show lower rates of LVSI and deep myometrial invasion [5]. However, another study that analyzed the relationship of molecular classification with tumor grade, stage, LVSI, myometrial invasion, adjuvant therapy, ethnicity and BMI did not determine any statistically significant relationships [23]. They reported that, although patients in the POLEmt group were at an advanced stage, they showed a better prognosis. Another study reported no cases of mortality in POLEmt patients in the year fol- lowing the diagnosis. A study determined POLEmt in only grade- 3 endometrioid carcinomas and did not determine POLE exonuclease domain mutations in serous, clear-cell and dedifferentiated carcinomas [23]. In the same study, P286R was the most common hotspot mutation, followed by V411L [23]. Another study determined Pro286Arg and Val411 Leu as the most common hotspot mutations [17]. In our study, $66.7 \%$ of patients with POLEmt were over 60 years of age. However, our study was performed on only high-grade endometrial carcinomas, and low-grade endometrial carcinomas were not included. In our study, $61.9 \%$ of POLEmt patients showed $>50 \%$ myometrial invasion, and the greatest myometrial invasion was seen in the $533 \mathrm{mt}$ group (70\%). Also, the most common hotspot mutation in our study was P436R, and only two of our patients showed co-occurring V411L and P436R hotspot mutations.

In the literature, the p53mt group is reported to be associated with more advanced stages. The p53mt group was reported to be associated with a more aggressive clinical course, to be more common in high-grade patients and in the presence of non-endometrioid histology, and to show higher rates of LVSI [4]. A study reported that the p53 group was almost exclusively associated with the serous subtype [3]. We determined in the present study that, in the p53mt group, the tumors were encountered at more advanced stages, typically in patients over 60 years of age, with a non-endometrioid histology, a tumor diameter larger than $2 \mathrm{~cm}$, more than $1 / 2$ myometrial invasion and higher rates of LVSI. In the literature, it was determined that, among the molecular groups, the POLEmt group had the best prognosis and the $533 \mathrm{~m}$ group had the poorest prognosis $[3,10]$. In agreement with the literature, our study also determined that the POLEmt group had the best prognosis and that the p53m group had the poorest prognosis.

\section{CONCLUSIONS}

In our study, we investigate the survival analysis histopathological and clinical prognostic parameters of patients with high grade endometrial carcinoma, based a new molecular classification. And in accordance with literature, we found that the $553 \mathrm{mt}$ group had the poorest prognosis and the POLEmt group had the best prognosis.

\section{Acknowledgments}

Not applicable.

\section{Funding}

Selçuk University Research Fund Accounting (BAP Project no: 19401103).

\section{Conflict of interest}

All authors declare no conflict of interest. 


\section{REFERENCES}

1. Miller KD, Siegel RL, Lin CC, et al. Cancer statistics, 2016. CA Cancer J Clin. 2016; 66(1): 7-30, doi: 10.3322/caac.21332, indexed in Pubmed: 26742998.

2. Gilks $C B$, Oliva E, Soslow RA. Poor interobserver reproducibility in the diagnosis of high-grade endometrial carcinoma. Am J Surg Pathol. 2013; 37(6): 874-881, doi: 10.1097/PAS.0b013e31827f576a, indexed in Pubmed: 23629444.

3. Bosse T, Nout RA, McAlpine JN, et al. Molecular classification of grade 3 endometrioid endometrial cancers identifies distinct prognostic subgroups. Am J Surg Pathol. 2018; 42(5): 561-568, doi: 10.1097/PAS.0000000000001020, indexed in Pubmed: 29505428.

4. Talhouk A, McConechy MK, Leung S, et al. Confirmation of ProMisE: A simple, genomics-based clinical classifier for endometrial cancer. Cancer. 2017; 123(5): 802-813, doi: 10.1002/cncr.30496, indexed in Pubmed: 28061006.

5. Cosgrove CM, Tritchler DL, Cohn DE, et al. An NRG Oncology/GOG study of molecular classification for risk prediction in endometrioid endometrial cancer. Gynecol Oncol. 2018; 148(1): 174-180, doi: 10.1016/j. ygyno.2017.10.037, indexed in Pubmed: 29132872.

6. Kommoss S, McConechy MK, Kommoss F, et al. Final validation of the ProMisE molecular classifier for endometrial carcinoma in a large population-based case series. Ann Oncol. 2018; 29(5): 1180-1188, doi: 10.1093/annonc/mdy058, indexed in Pubmed: 29432521.

7. Proctor L, Pradhan M, Leung S, et al. Assessment of DNA Ploidy in the ProMisE molecular subgroups of endometrial cancer. Gynecol Oncol. 2017; 146(3): 596-602, doi: 10.1016/j.ygyno.2017.06.020, indexed in Pubmed: 28647100.

8. Stelloo E, Bosse T, Nout RA, et al. Refining prognosis and identifying targetable pathways for high-risk endometrial cancer; a TransPORTEC initiative. Mod Pathol. 2015; 28(6): 836-844, doi: 10.1038/modpathol.2015.43, indexed in Pubmed: 25720322.

9. Stelloo E, Nout RA, Osse EM, et al. Improved risk assessment by integrating molecular and clinicopathological factors in early-stage endometrial cancer-combined analysis of the PORTEC cohorts. Clin Cancer Res. 2016; 22(16): 4215-4224, doi: 10.1158/1078-0432.CCR-15-2878, indexed in Pubmed: 27006490.

10. Talhouk A, Hoang LN, McConechy MK, et al. Molecular classification of endometrial carcinoma on diagnostic specimens is highly concordant with final hysterectomy: Earlier prognostic information to guide treatment. Gynecol Oncol. 2016; 143(1): 46-53, doi: 10.1016/j.ygyno.2016.07.090, indexed in Pubmed: 27421752.

11. DeSantis CE, Ma J, Gaudet MM, et al. Cancer statistics, 2019. CA Cancer J Clin. 2019; 69(1): 7-34, doi: 10.3322/caac.21551, indexed in Pubmed: 30620402.

12. Hoang LN, Kinloch MA, Leo JM, et al. Interobserver agreement in endometrial carcinoma histotype diagnosis varies depending on The Cancer
Genome Atlas (TCGA)-based Molecular Subgroup. Am J Surg Pathol. 2017; 41(2): 245-252, doi: 10.1097/PAS.0000000000000764, indexed in Pubmed: 28079598.

13. McAlpine JN, Temkin SM, Mackay HJ. Endometrial cancer: Not your grandmother's cancer. Cancer. 2016; 122(18): 2787-2798, doi: 10.1002/cncr.30094, indexed in Pubmed: 27308732.

14. Guan H, Semaan A, Bandyopadhyay S, et al. Prognosis and reproducibility of new and existing binary grading systems for endometrial carcinoma compared to FIGO grading in hysterectomy specimens. Int J Gynecol Cancer. 2011; 21(4): 654-660, doi: 10.1097/IGC.0b013e31821454f1, indexed in Pubmed: 21543931.

15. Bendifallah $S$, Canlorbe $G$, Collinet $P$, et al. Just how accurate are the major risk stratification systems for early-stage endometrial cancer? Br J Cancer. 2015; 112(5): 793-801, doi: 10.1038/bjc.2015.35, indexed in Pubmed: 25675149.

16. Murali R, Soslow RA, Weigelt B. Classification of endometrial carcinoma: more than two types. Lancet Oncol. 2014; 15(7): e268-e278, doi: 10.1016/S1470-2045(13)70591-6, indexed in Pubmed: 24872110.

17. Hussein YR, Weigelt B, Levine DA, et al. Clinicopathological analysis of endometrial carcinomas harboring somatic POLE exonuclease domain mutations. Mod Pathol. 2015; 28(4): 505-514, doi: 10.1038/modpathol.2014.143, indexed in Pubmed: 25394778.

18. Nelson BH, McAlpine JN. The more tumors change, the more they stay tame: do T cells keep POLE ultramutated endometrial carcinomas in check? Gynecol Oncol. 2015; 138(1): 1-2, doi: 10.1016/j. ygyno.2015.06.004, indexed in Pubmed: 26072691.

19. Briggs $S$, Tomlinson I. Germline and somatic polymerase $\varepsilon$ and $\delta$ mutations define a new class of hypermutated colorectal and endometrial cancers. J Pathol. 2013; 230(2): 148-153, doi: 10.1002/path.4185, indexed in Pubmed: 23447401.

20. McAlpine J, Leon-Castillo A, Bosse T. The rise of a novel classification system for endometrial carcinoma; integration of molecular subclasses. J Pathol. 2018; 244(5): 538-549, doi: 10.1002/path.5034, indexed in Pubmed: 29344951.

21. Karnezis AN, Hoang LN, Coatham M, et al. Loss of switch/sucrose non-fermenting complex protein expression is associated with dedifferentiation in endometrial carcinomas. Mod Pathol. 2016; 29(3): 302-314 doi: 10.1038/modpathol.2015.155, indexed in Pubmed: 26743474.

22. Travaglino A, Raffone A, Stradella $C$, et al. Impact of endometrial carcinoma histotype on the prognostic value of the TCGA molecular subgroups. Arch Gynecol Obstet. 2020; 301(6): 1355-1363, doi: 10.1007/s00404-020-05542-1, indexed in Pubmed: 32296930.

23. Meng Bo, Hoang LN, Mclntyre JB, et al. POLE exonuclease domain mutation predicts long progression-free survival in grade 3 endometrioid carcinoma of the endometrium. Gynecol Oncol. 2014; 134(1): 15-19, doi: 10.1016/j.ygyno.2014.05.006, indexed in Pubmed: 24844595. 